BULL. AUSTRAL, MATH. SOC.

VOL. 29 (1984), 401-404.

\title{
FREE PRODUCTS OF LOCALLY INDICABLE GROUPS WITH A SINGLE RELATOR
}

\author{
BENJAMIN BAUMSLAG
}

\begin{abstract}
The method of proof of Magnus introduced in 1930 is adapted to prove the following theorem of Howie. If $A$ and $B$ are groups for which every finitely generated subgroup has an infinite cyclic image, and if one adds an additional relation (with obvious exceptions), then in the resultant group both A and B appear isomorphically.
\end{abstract}

We recall that a group is said to be locally indicable if every finitely generated subgroup has an infinite cyclic homomorphic image. For convenience, if $R$ is an element of a group $G, G / R$ will denote $G / N$ where $N$ is the normal closure of $R$ in $G$.

THEOREM 1. Let $\mathrm{A}$ and $\mathrm{B}$ be locally indicable groups and let $G=(A * B) / R$, where $R$ is a cyclically reduced word of length at least 2 . Then the canonical maps $\mathrm{A} \rightarrow \mathrm{G}, \mathrm{B} \rightarrow \mathrm{G}$ are injective.

This theorem, which is due to Howie [2] (see also references in his paper to the work of Brodskii and Short), is a generalisation of the Freiheitsatz of [3]. It is the object of this note to prove Theorem 1 using combinatorial methods like those of Magnus [3], in the style of [1].

Proof of Theorem 1. We argue by induction on the length $\lambda$ of $R$. Let $A_{0}$ (respectively $B_{0}$ ) denote the subgroup of $A$ (respectively $B$ ) generated by those elements which appear in $R$. Then $R$ regarded as a word in $A_{0}{ }^{*} B_{0}$ is cyclically reduced of length $\lambda$. Let $G_{0}=A_{0}{ }^{*} B_{0} / R$. Received 5 January 1984

Copyright Clearance Centre, Inc. Serial-fee code: 0004-9727/84 $\$ A 2.00+0.00$ 
If we can show that the canonical maps of $A_{0}$ and $B_{0}$ into $G_{0}$ are injective, it will follow that

$$
G \cong A \quad \stackrel{A}{A_{0}^{*}} \quad G_{0} \quad \stackrel{B_{0}^{*}}{B_{0}}
$$

so that the canonical maps of $A$ and $B$ into $G$ will also be injective. Hence we may assume, without loss of generality, that $A=A_{0}$ and $B=B_{0}$. In particular, if $\lambda=2$ (the initial case of the induction), then $A$ and $B$ are infinite cyclic groups and the result holds. Since $A=A_{0}$ and $B={ }^{B}{ }^{\prime}$ both are finitely generated, and hence each has an infinite cyclic image. By symmetry, it suffices to show that the canonical map of $A$ into $G$ is injective.

Case 1. $\quad B=\langle b\rangle$ is infinite cyclic.

Let $D=\langle d\rangle$ be infinite cyclic. Then there is an epimorphism of $A$ onto $D$ with kernel $N$ say and with $A / N=\langle a N\rangle$. This epimorphism induces a homomorphism of $A{ }^{*} B$ onto $D * B$, and suppose that under this homomorphism $R$ is mapped to $R_{l}$.

Subcase $A$. Suppose that d occurs with zero exponent sum in $R_{1}$.

Thus $R$ lies in the normal closure in $A{ }^{*} B$ of $N \cup B$. Put $b_{i}=a^{-i} b^{i}$ and write $R$ in terms of the $b_{i}$ and the elements of $N$. At least two of the $b_{i}$ must be involved in expressing $R$, since $R$ is of length at least 4 and some $a^{r} n$ ( $r$ a non-zero integer and $n \in N$ ) must occur in $R$ by our assumption that $A=A_{0}$.

Let $s$ be the least suffix of $a b_{i}$ appearing in $R$ and let $t$ be largest. Let

$$
\begin{gathered}
K=N *\left\langle b_{s}\right\rangle *\left\langle b_{s+1}\right\rangle * \ldots *\left(b_{t}\right\rangle, K_{0}=N^{*}\left(b_{s}\right\rangle *\left\langle b_{s+1}\right\rangle * \ldots *\left\langle b_{t-1}\right\rangle \\
\text { and } K_{1}=N *\left(b_{s+1}\right\rangle *\left\langle b_{s+2}\right\rangle * \ldots *\left\langle b_{t}\right\rangle .
\end{gathered}
$$

$R$ is of length smaller than $\lambda$ as a word in the free product $\mathrm{K}_{0} \star\left\langle\mathrm{b}_{t}\right\rangle$. Hence by the induction hypothesis, $\mathrm{K}_{0}$ is embedded in $\overline{\mathrm{K}}=\mathrm{K} / \mathrm{R}$. Similarly, $\mathrm{R}$ as a word in the free product $\left\langle\mathrm{b}_{\mathrm{S}}\right\rangle * \mathrm{~K}_{1}$ is of length less than $\lambda$ and so $K_{1}$ is embedded in $\bar{K}$. Thus $G$ is the HNN 
extension of $\bar{K}$ with free element $a$ and associated subgroups $K_{0}$ and $\mathrm{K}_{1}$, with a taking $\mathrm{b}_{i}$ to $\mathrm{b}_{i+1}(s \leqslant i<t)$, and acting on the elements of $\mathrm{N}$ by conjugation in $\mathrm{A}$. Thus $\mathrm{N}$ and hence $\mathrm{A}$ is embedded in $\mathrm{G}$.

Subcase B. Suppose $d$ appears with non-zero exponent sum $\delta$ in $R_{1}$ and $b$ appears with non-zero exponent sum $B$.

Adjoin a $B$-th root $\hat{a}$ of a to obtain the group $\hat{A}$ and a $\beta-t h$ root $\hat{a}$ of $d$ to obtain the infinite cyclic group $\hat{D}$. Then $\hat{A}$ is again locally indicable (as can be shown using the subgroup theorem for a free product with an amalgamation) and there is an obvious homomorphism $a: \hat{A} \rightarrow \hat{D}$ which takes $\hat{a}$ to $\hat{d}$ and $a$ to $d$. Let $\hat{b}=\hat{b} a \delta$ and $B=\langle\hat{b}\rangle$. Then it is easy to check that $\hat{A} * B=\hat{A} * \hat{B}$ by using the homomorphism definition of a free product. It is also clear that in the image of $R$ under the homomorphism induced by $\alpha$ from $\hat{A} * \hat{B}$ into $\hat{D} * \hat{B}$, that $\hat{d}$ appears with zero exponent sum. We can then apply subcase $A$.

Case 2. B arbitrary.

Then there exists an epimorphism $\phi$ of $B$ onto an infinite cyclic group $C=(C)$. This induces a homomorphism of $A * B$ onto $A * C$. Let $R_{2}$ be the image of $R$ under this map. If $C$ appears with non-zero exponent sum in $R_{2}$, then if the length of $R_{2}$ is 1 , then $A * C / R_{2}$ is the free product of $A$ and a finite cyclic group and hence $A$ is embedded in it, which implies the required result.

Otherwise if $R_{2}$ is of length at least 2 , then we can use case 1 to establish that $A$ is embedded in $A * C / R_{2}$, and hence the result.

So we are left with the case that $C$ appears with zero exponent sum in $R_{2}$ which is itself of length at least two. This is the case dealt with in the last five paragraphs of page 173 of [2]; we repeat this argument for completeness.

Let $K$ be the kernel of $\phi$ and let $b$ be a pre-image of $c$ under $\phi$. Then $R$ lies in the normal closure of $A$ and $K$ which has the form $L=\left({ }^{*} A_{i} \mid i\right.$ in the integers $) * K$, where $A_{i}=b^{-i} A b^{i}$. 
Since $B=B_{0}$ it follows that at least two of the groups $A_{i}$ are involved in $R$. Suppose $S$ is smallest index such that $A_{S}$ is involved in $R$, and $t$ is the largest such index.

Define $K_{0}=A_{S+1}{ }^{*} \ldots{ }^{*} A_{t}{ }^{*}$ and $K_{1}=A_{s} * \ldots * A_{t-1}{ }^{*} K$. Then $\mathrm{R}$ belongs to $\mathrm{L}_{0}=\mathrm{A}_{\mathrm{S}}{ }^{{ }_{\mathrm{K}}} \mathrm{K}_{0}=\mathrm{A}_{\mathrm{t}}{ }^{*} \mathrm{~K}_{1}$, and all the groups $\mathrm{A}_{\mathrm{s}}, \mathrm{A}_{\mathrm{t}}, \mathrm{K}_{0}, \mathrm{~K}_{1}$ are locally indicable. Let $G_{2}=L_{0} / R$.

Let $\lambda_{2}$ denote the length of some cyclically reduced conjugate of $\mathrm{R}$ regarded as a word in $A_{S}{ }^{*} K_{0}$. Then $\lambda_{2} \leqslant \lambda_{1} \leqslant \lambda$. If $\lambda_{2}=\lambda$, then $s=t=0$, and $R$ belongs to $A^{*} K$ which contradicts $B=B_{0}$. If $\lambda_{2} \leqslant 1$, then $R$ is conjugate in $A_{S}{ }^{\star} K_{0}$ to an element of $A_{S}$ or $K_{0}$. The first contradicts the fact that $\lambda \geqslant 2$, the second the choice of $s$. Hence $2 \leqslant \lambda_{2}<\lambda$.

By the inductive hypothesis, the canonical maps of $A_{S}$ and $K_{0}$ into $G_{2}$ are injective. The result now follows since $G$ is the HNN extension of $G_{2}$ with $b$ conjugating $k_{1}$ onto $K_{0}$.

\section{References}

[1] B. Baumslag and S.J. Pride, "An extension of the Freiheitsatz", Math. Proc. Combridge Phiz. Soc. 89 (1981), 35-41.

[2] J. Howie, "On pairs of 2-complexes and systems of equations over groups", J. reine angewandte Math. 324 (1981), 165-174.

[3] W. Magnus, "Ueber diskontinuierliche Gruppen mit einer definierenden Relation (Der Freiheitsatz)", J. reine angew. Math. 163 (1930), 141-165.

Department of Mathematics,

Imperial College of Science \& Technology,

Queen's Gate,

London SW7 2BZ

England 